\title{
Double-Diffusion MHD Free Convective Flow Along a Sphere in the Presence of a Homogeneous Chemical Reaction and Soret and Dufour Effects
}

\author{
A. J. Chamkha ${ }^{1,2}$, A. M. Aly ${ }^{3}$, Z. A. S. Raizah $^{4}$ \\ ${ }^{1}$ Mechanical Engineering Department, Prince Mohammad Bin Fahd University, Al-Khobar, Saudi Arabia \\ ${ }^{2}$ Prince Sultan Endowment for Energy and Environment, Prince Mohammad Bin Fahd University, Al-Khobar, Saudi Arabia \\ ${ }^{3}$ Department of Mathematics, Faculty of Science, South Valley University, Qena, Egypt \\ ${ }^{4}$ Department of Mathematics, Faculty of Science for Girls, Abha, King Khalid University, Asir, Saudia Arabia
}

Email address:

abdelreheam.abdallah@sci.svu.edu.eg (A. M. Aly)

\section{To cite this article:}

A. J. Chamkha, A. M. Aly, Z. A. S. Raizah. Double-Diffusion MHD Free Convective Flow Along a Sphere in the Presence of a Homogeneous Chemical Reaction and Soret and Dufour Effects. Applied and Computational Mathematics. Vol. 6, No. 1, 2017 , pp. $34-44$. doi: $10.11648 /$ j.acm.20170601.12

Received: January 5, 2017; Accepted: January 19, 2017; Published: February 23, 2017

\begin{abstract}
A numerical solution is presented for the effects of chemical reaction, thermal radiation, Soret number, Dufour number and magnetic field on double-diffusion free convection flow along a sphere. The governing boundary-layer equations of the problem are formulated and transformed into non-similar form. The obtained equations are solved numerically by an efficient, iterative, tri-diagonal, implicit finite-difference method. The Roseland approximation is used to describe the radiative heat flux in the energy equation. Representative results for the fluid velocity, temperature and solute concentration profiles as well as the local heat and mass transfer rates for various values of the physical parameters are displayed in both graphical and tabular forms.
\end{abstract}

Keywords: Chemical Reaction, Radiation, Double Diffusion, MHD Flow, Soret and Dufour Effects

\section{Introduction}

Double-diffusive convection which is a consequence of buoyancy effects caused by diffusion of heat and chemical species finds applications in a variety of engineering processes such as heat exchanger devices, petroleum reservoirs, chemical catalytic reactors and processes, nuclear waste repositories and others. Flows of electricallyconducting fluids in the presence of a magnetic field have received much attention by many researchers in recent years due to their importance from technical point of view. In addition, the study of heat and mass transfer processes is useful for improving a number of chemical technologies such as polymer production and food processing. In nature, the presence of pure air or water is impossible as some foreign species (possibly chemically reacting) may be present either naturally or intentionally mixed with air or water.

Chemical reactions can be classified as either heterogeneous or homogeneous processes. This depends on whether they occur at an interface or as a single phase volume reaction. In well-mixed systems, the reaction is heterogeneous if it takes place at an interface and homogeneous if it takes place in solution. In most cases of chemical reactions, the reaction rate depends on the concentration of the species itself.

We are particularly interested in cases in which diffusion and chemical reaction occur at roughly the same speed. When diffusion is much faster than chemical reaction, then only chemical factors influence the chemical reaction rate; and when diffusion is not much faster than chemical reaction, the diffusion and kinetics interact to produce very different effects. Anjalidevi and Kandasamy $[1,2]$ have analyzed the effects of chemical reaction, heat and mass transfer on laminar flow with or without Magnetohydrodynamics (MHD) effects along a semi infinite horizontal plate. Chamkha et al. [3] investigated numerically the effects of chemical reaction, thermal radiation and heat generation or 
absorption on unsteady free convective heat and mass transfer along an infinite vertical porous plate in the presence of a transverse magnetic field and Hall current. Chamkha et al. [4] analyzed the similarity solution for unsteady heat and mass transfer from a stretching surface embedded in a porous medium with suction/injection and chemical reaction effects. Muthucumaraswamy and Ganesan [5,6] have studied the impulsive motion of a vertical plate with heat flux, mass flux, suction and diffusion of chemically reactive species. The flow and mass transfer on a stretching sheet with a magnetic field and chemically reactive species were examined by Takhar et al. [7]. Muthucumaraswamy [8] has analyzed the effects of a chemical reaction on a moving isothermal vertical surface with suction.

The mass transfer caused by temperature gradient is called the Soret effect and it has been utilized for isotope separation and in a mixture between gases with very light molecular weight $\left(\mathrm{H}_{2}, \mathrm{He}\right)$ and of medium molecular weight $\left(\mathrm{H}_{2}\right.$, air). Also, the heat transfer caused by concentration gradient is called the Dufour effect. Weaver et a1. [9] have pointed out that when the differences of the temperature and the concentration are large or when the difference of the molecular mass of the two elements in a binary mixture is great, the coupled interaction is significant. A primary discussion on the effect of the cross-coupled diffusion in a system with horizontal temperature and concentration a gradient was made by Malashetty and Gaikwad [10]. Thermal diffusion, also called solutal thermo-diffusion or Soret effect [11], corresponds to species differentiation developing in an initially homogeneous mixture submitted to a thermal gradient. In many studies, Dufour and Soret effects are neglected on the basis that they are of a smaller order of magnitude than the effects described by Fourier's and Fick's laws. There are, however, exceptions. Eckert and Drake [12] have presented several cases when the Dufour effect cannot be neglected. Kafoussias and Williams [13] considered the boundary-layer flows in the presence of Soret and Dufour effects associated with thermal diffusion and diffusion thermo for the mixed forced-natural convection. Mansour et al. [14] studied the effects of chemical reaction, thermal stratification, Soret number and Dufour number on MHD free convective heat and mass transfer of a viscous, incompressible and electrically conducting fluid on a vertical stretching surface embedded in a saturated porous medium. Chamkha and Aly [15] studied numerically the boundarylayer stagnation-point flow of a polar fluid towards a stretching surface embedded in porous media in the presence of the effects of Soret and Dufour numbers and first-order homogeneous chemical reaction.

For some industrial applications such as glass production and furnace design and in space technology applications such as cosmical flight aerodynamics rocket, propulsion systems, plasma physics and spacecraft re-entry aerothermodynamics which operate at higher temperatures, radiation effects can be significant. When radiation is taken into account, the governing equations become quite complicated and hence, many difficulties arise while solving such equations. However, Greif et al. [16] have shown that in the optically thin limit, the physical situation can be simplified and then they have derived an exact solution to fully-developed vertical channel flow for a radiative fluid. Cogley et al. [17] have shown that for an optically thin limit, the fluid does not absorb its own emitted radiation or there is no selfabsorption, but the fluid does absorb radiation emitted by the boundaries.

Convective flows along circular geometries and spheres have been considered by many previous authors due to their industrial applications. Molla et al. [18] have investigated MHD natural convection flow on a sphere in presence of heat generation. The problems of free convection boundary layer flow over or on bodies of various shapes discussed by many researchers. Amongst them, Nazar et al. [19, 20] have considered free convection boundary-layer on an isothermal sphere and on an isothermal horizontal circular cylinder in a micropolar fluid. Mansour et al. [21] have examined the heat and mass transfer in magnetohydrodynamic flow of micropolar fluid on a circular cylinder with uniform heat and mass flux. Alam et al. [22] have studied the effects of viscous dissipation on MHD natural convection flow along a sphere. Huang and Chen [23] have solved the laminar free convection flow about a sphere with surface blowing and suction effects. Cheng [24] has studied natural convection heat and mass transfer from a sphere in micropolar fluids with constant wall temperature and concentration.

Recently, Chamkha et al. [25-28] studied free convective flows along vertical and stretching cones, sphere and a vertical cylinder embedded in porous media under the effects of different physical properties. Khan and sultan [29] studied the double diffusive Darcian convection flow of EyringPowell fluid from a cone embedded in a homogeneous porous medium with the effects of Soret and Dufour. Raju et al. [30] performed numerical investigations using mixing of copper nanoparticles in the kerosene to study the effects of temperature dependent viscosity, heat source/sink and viscous dissipation on natural convective heat transfer of radiative magnetohydrodynamic (MHD) non-Newtonian nanofluid caused by a cone.

In the present work, a numerical solution is presented for coupled heat and mass transfer by natural convection MHD flow along a sphere in the presence of Soret, Dufour, thermal radiation and chemical reaction effects.

\section{Mathematical Analysis}

Consider steady, two-dimensional, MHD double-diffusion convective boundary-layer flow of an incompressible, viscous and electrically-conducting fluid along a sphere in the presence of a uniform magnetic field and a homogeneous chemical reaction and considering Soret and Dufour effects. The fluid properties are assumed to be constant and the chemical reaction is taking place in the flow. The physical properties and rate of chemical reaction $k_{c}$ are constant throughout the fluid. Under the boundary-layer assumptions and considering the Boussinesq approximation, the governing equations are given by: 


$$
\begin{gathered}
\frac{\partial}{\partial x}(r u)+\frac{\partial}{\partial y}(r v)=0 \\
u \frac{\partial u}{\partial x}+v \frac{\partial u}{\partial y}=v \frac{\partial^{2} u}{\partial y^{2}}+\left[g \beta_{T}\left(T-T_{\infty}\right)+g \beta_{C}\left(C-C_{\infty}\right)\right] \sin \left(\frac{x}{a}\right)-\frac{\sigma B_{0}^{2}}{\rho} u \\
u \frac{\partial T}{\partial x}+v \frac{\partial T}{\partial y}=\frac{k}{\rho C_{P}} \frac{\partial^{2} T}{\partial y^{2}}-\frac{1}{\rho C_{P}} \frac{\partial q_{r}}{\partial y}+\frac{D_{m} k_{T}}{C_{S} C_{P}} \frac{\partial^{2} C}{\partial y^{2}} \\
u \frac{\partial C}{\partial x}+v \frac{\partial C}{\partial y}=D_{m} \frac{\partial^{2} C}{\partial y^{2}}-k_{c}\left(C-C_{\infty}\right)+\frac{D_{m} k_{T}}{T_{M}} \frac{\partial^{2} T}{\partial y^{2}}
\end{gathered}
$$

where $\mathrm{x}, \mathrm{y}$ and $r=r(x)$ are the Cartesian coordinates and the radial distance from the symmetrical axis to the surface of the sphere, respectively. $u, v, T$ and $C$ are the fluid $\mathrm{x}-$ component of velocity, y-component of velocity, temperature and solute concentration, respectively. $\sigma$ is the electric conductivity, $B_{0}$ is the strength of magnetic field, $\rho$ is the density, $v$ is the kinematic viscosity, $k$ is the fluid thermal conductivity, $C_{P}$ is the specific heat at constant pressure and $q_{r}$ is the radiative heat flux. $k_{c}$ is the rate of chemical reaction, $g$ is the acceleration due to gravity, $\beta_{T}$ is the volumetric coefficient of thermal expansion, $\beta_{C}$ is the volumetric coefficient of concentration expansion. $D_{m}$ is coefficient of mass diffusivity. $T_{\infty}$ and $C_{\infty}$ are the free stream temperature and concentration, respectively. $T_{m}$ is the mean fluid temperature, $k_{T}$ is the thermal diffusion ratio and $C_{S}$ is the concentration susceptibility.

The physical boundary conditions for the problem are:

$$
\begin{aligned}
& u=v=0, T=T_{\infty}, C=C_{\infty}, \text { on } y=0 \\
& u \rightarrow 0, T \rightarrow T_{\infty}, \quad C \rightarrow C_{\infty} \text { as } y \rightarrow \infty
\end{aligned}
$$

The radial distance from the symmetrical axis to the surface of the sphere $r=r(x)$ is given by

$$
r=a \sin \left(\frac{x}{a}\right)
$$

where $a$ is the radius of the sphere. The current physical model with coordinate system has been shown in Figure 1.

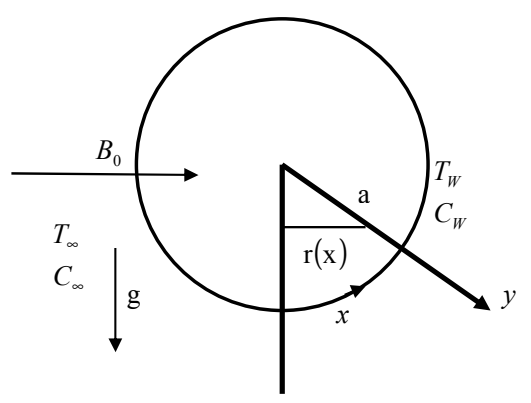

Figure 1. The physical model and coordinate system.
The radiative heat flux term is given and simplified by using the Rosseland approximation (see Sparrow and Cess [31]) as

$$
q_{r}=-\frac{4 \sigma_{0}}{3 k^{*}} \frac{\partial T^{4}}{\partial y}
$$

where $\sigma_{0}$ and $k^{*}$ are Stefan-Boltzman constant and mean absorption coefficient, respectively. The Taylor series expansion for $T^{4}$ neglecting higher order terms gives

$$
T^{4}=4 T_{\infty}^{3} T-3 T_{\infty}^{4},
$$

Using Equations (7) and (8) in the energy equation (3), one obtains

$$
u \frac{\partial T}{\partial x}+v \frac{\partial T}{\partial y}=\frac{v}{\operatorname{Pr}}\left(1+\frac{4}{3 R}\right) \frac{\partial^{2} T}{\partial y^{2}}+\frac{D_{m} k_{T}}{C_{S} C_{P}} \frac{\partial^{2} C}{\partial y^{2}},
$$

where $\operatorname{Pr}=\frac{\rho v C_{P}}{k}$ is the Prandtl number and $R=\frac{k k^{*}}{4 \sigma T_{\infty}^{3}}$ is the radiation parameter.

Substituting the following dimensionless variables:

$$
\begin{gathered}
\xi=\frac{x}{a}, \eta=G r^{1 / 4} \frac{y}{a}, U=\frac{a}{v} G r^{-1 / 2} u, V=\frac{a}{v} G r^{-1 / 4} v \\
\theta=\frac{T-T_{\infty}}{T_{W}-T_{\infty}}, \varphi=\frac{C-C_{\infty}}{C_{W}-C_{\infty}}
\end{gathered}
$$

where $G r=\frac{g \beta_{T}\left(T_{W}-T_{\infty}\right) a^{3}}{v^{2}}$ is the Grashof number into Equations (1), (2), (4) and (9) yields the following dimensionless equations:

$$
\begin{gathered}
\frac{\partial}{\partial \xi}(r U)+\frac{\partial}{\partial \eta}(r V)=0 \\
U \frac{\partial U}{\partial \xi}+V \frac{\partial U}{\partial \eta}=\frac{\partial^{2} U}{\partial \eta^{2}}+(\theta+N \varphi) \sin \xi-M U \\
U \frac{\partial \theta}{\partial \xi}+V \frac{\partial \theta}{\partial \eta}=\frac{1}{\operatorname{Pr}}\left(1+\frac{4}{3 R}\right) \frac{\partial^{2} \theta}{\partial \eta^{2}}+D u \frac{\partial^{2} \varphi}{\partial \eta^{2}} \\
U \frac{\partial \varphi}{\partial \xi}+V \frac{\partial \varphi}{\partial \eta}=\frac{1}{S c} \frac{\partial^{2} \varphi}{\partial \eta^{2}}-\gamma \varphi+S r \frac{\partial^{2} \theta}{\partial \eta^{2}}
\end{gathered}
$$

where $M=\frac{\sigma B_{0}^{2} a^{2}}{\rho \nu G r^{1 / 2}}$ is the magnetic field parameter, $D u=\frac{D_{m} k_{T}}{v C_{S} C_{P}}\left(\frac{C_{w}-C_{\infty}}{T_{w}-T_{\infty}}\right)$ is the Dufour number, $S r=\frac{D_{m} k_{T}}{v T_{m}}\left(\frac{T_{w}-T_{\infty}}{C_{w}-C_{\infty}}\right)$ is the Soret number and $\gamma=\frac{k_{c} a^{2}}{v G r^{1 / 2}}$ is the chemical reaction parameter.

The dimensionless boundary conditions become: 


$$
\begin{gathered}
U=V=0, \theta=\varphi=1 \text { at } \eta=0 \\
U \rightarrow 0, \theta \rightarrow 0, \varphi \rightarrow 0 \text { at } \eta \rightarrow \infty
\end{gathered}
$$$$
\psi(\xi, \eta)=\xi r(\xi) f(\xi, \eta),
$$

and applying it to the above dimensionless equations yields

Assuming that

$$
\begin{gathered}
\frac{\partial^{3} f}{\partial \eta^{3}}+\left(1+\frac{\xi}{\sin \xi} \cos \xi\right) f \frac{\partial^{2} f}{\partial \eta^{2}}-\left(\frac{\partial f}{\partial \eta}\right)^{2}-M \frac{\partial f}{\partial \eta}+\frac{\sin \xi}{\xi}(\theta+N \varphi)=\xi\left(\frac{\partial f}{\partial \eta} \frac{\partial^{2} f}{\partial \eta \partial \xi}-\frac{\partial f}{\partial \xi} \frac{\partial^{2} f}{\partial \eta^{2}}\right) \\
\frac{1}{\operatorname{Pr}}\left(1+\frac{4}{3 R}\right) \frac{\partial^{2} \theta}{\partial \eta^{2}}+\left(1+\frac{\xi \cos \xi}{\sin \xi}\right) f \frac{\partial \theta}{\partial \eta}+D u \frac{\partial^{2} \varphi}{\partial \eta^{2}}=\xi\left(\frac{\partial f}{\partial \eta} \frac{\partial \theta}{\partial \xi}-\frac{\partial f}{\partial \xi} \frac{\partial \theta}{\partial \eta}\right) \\
\frac{1}{S c} \frac{\partial^{2} \varphi}{\partial \eta^{2}}+\left(1+\frac{\xi \cos \xi}{\sin \xi}\right) f \frac{\partial \varphi}{\partial \eta}-\gamma \varphi+S r \frac{\partial^{2} \theta}{\partial \eta^{2}}=\xi\left(\frac{\partial f}{\partial \eta} \frac{\partial \varphi}{\partial \xi}-\frac{\partial f}{\partial \xi} \frac{\partial \varphi}{\partial \eta}\right)
\end{gathered}
$$

The corresponding transformed boundary conditions are:

$$
\begin{aligned}
& f=f^{\prime}=0, \theta=\varphi=1 \quad \text { at } \eta=0 \\
& f^{\prime} \rightarrow 0, \theta \rightarrow 0, \varphi \rightarrow 0 \text { at } \eta \rightarrow \infty
\end{aligned}
$$

It can be seen that at the lower stagnation point of the sphere $(\xi \approx 0)$ the above equations and conditions reduce to the following ordinary differential equations:

$$
\begin{gathered}
\frac{\partial^{3} f}{\partial \eta^{3}}+2 f \frac{\partial^{2} f}{\partial \eta^{2}}-\left(\frac{\partial f}{\partial \eta}\right)^{2}+(\theta+N \varphi)-M \frac{\partial f}{\partial \eta}=0 \\
\frac{1}{\operatorname{Pr}}\left(1+\frac{4}{3 R}\right) \frac{\partial^{2} \theta}{\partial \eta^{2}}+D u \frac{\partial^{2} \varphi}{\partial \eta^{2}}+2 f \frac{\partial \theta}{\partial \eta}=0 \\
\frac{1}{S c} \frac{\partial^{2} \varphi}{\partial \eta^{2}}-\gamma \varphi+S r \frac{\partial^{2} \theta}{\partial \eta^{2}}+2 f \frac{\partial \varphi}{\partial \eta}=0
\end{gathered}
$$

subject to the boundary conditions

$$
\begin{aligned}
f=f^{\prime}=0, \theta=\varphi=1 \quad \text { at } & \eta=0 \\
f^{\prime} \rightarrow 0, \theta & \rightarrow 0, \varphi \rightarrow 0 \quad \text { at } \quad \eta \rightarrow \infty
\end{aligned}
$$

Of special significance for this type of flow and heat and mass transfer situation are the skin-friction coefficient $\mathrm{C}_{\mathrm{f}}$, the heat transfer rate or Nusselt number $\mathrm{Nu}$ and the mass transfer rate or Sherwood number Sh. These physical quantities are defined in dimensionless form, respectively, as follows:

$$
\begin{gathered}
C_{f}=\frac{G r^{-3 / 4} a^{2}}{\mu v} \tau_{w}, N u=\frac{a G r^{-1 / 4}}{k\left(T_{w}-T_{\infty}\right)} q_{w} \text { and } \\
S h=\frac{a G r^{-1 / 4}}{\rho D_{m}\left(C_{w}-C_{\infty}\right)} m_{w}
\end{gathered}
$$

where $\quad \tau_{w}=\mu\left(\frac{\partial u}{\partial y}\right)_{y=0}, \quad q_{w}=-k\left(\frac{\partial T}{\partial y}\right)_{y=0} \quad$ and $m_{w}=-\rho D_{m}\left(\frac{\partial C}{\partial y}\right)_{y=0}$ are the shear stress, surface heat flux and the surface mass flux, respectively. Using the new variables (10) and (16) gives

$$
\begin{gathered}
C_{f}=\xi\left(\frac{\partial^{2} f}{\partial \eta^{2}}\right)_{\eta=0}, N u=\left(1+\frac{4}{3 R}\right)\left[-\theta^{\prime}(\eta)\right]_{\eta=0}, \\
S h=\left[-\varphi^{\prime}(\eta)\right]_{\eta=0}
\end{gathered}
$$

\section{Numerical Method}

The non-similar equations (17) through (19) are linearized and then descritized using three points central difference quotients with variable step sizes in the $\eta$ direction and using two-point backward difference formulae in the $\xi$ direction with a constant step size. The resulting equations form a tridiagonal system of algebraic equations that can be solved by the well known Thomas algorithm (see Blottner [32]). The solution process starts at $\xi=0$ where Equations (21) through (23) are solved and then marches forward using the solution at the previous line of constant $\xi$ until it reaches the desired value of $\xi\left(=120^{\circ}\right.$ in this case). Due to the nonlinearities of the equations, an iterative solution with successive over or under relaxation techniques is required. The convergence criterion required that the maximum absolute error between two successive iterations be $10^{-6}$. The computational domain was made of 196 grids in the $\eta$ direction and 1000 grids in the $\xi$ direction. A starting step size of 0.001 in the $\eta$ direction with an increase of 1.0375 times the previous step size and a constant step size in the $\xi$ direction of 0.0021 were found to give very accurate results. The maximum value of $\eta\left(\eta_{\infty}\right)$ which represented the ambient conditions was assumed to be 35 . The step sizes employed were arrived at after performing numerical experimentations to assess grid independence and ensure accuracy of the results. The accuracy of the aforementioned numerical method was validated by direct comparisons with the numerical results reported earlier by Huang and Chen [23] and Cheng [24] in the absence of magnetic field, thermal radiation, Soret and Dufour and 
concentration buoyancy effects. Table 1 presents the results of this comparison. It can be seen from this table that excellent agreement between the results exists. This favorable comparison lends confidence in the numerical results to be reported in the next section.

Table 1. Comparison of values of $\mathrm{Nu} / \mathrm{Gr}^{1 / 4}$ with Huang and Chen [23] and Cheng [24] for $D u=0, M=0, N=0, P r=0.7, R=\infty$ and $S r=0$.

\begin{tabular}{llll}
\hline$\xi$ & Huang and Chen [23] & Cheng [24] & Present work (Nu) \\
\hline 0 & 0.4574 & 0.4576 & 0.4577 \\
0.1745 & 0.4563 & 0.4565 & 0.4564 \\
0.3491 & 0.4532 & 0.4534 & 0.4533 \\
0.5236 & 0.4480 & 0.4481 & 0.4480 \\
0.6981 & 0.4407 & 0.4407 & 0.4407 \\
0.8727 & 0.4312 & 0.4310 & 0.4312 \\
1.047 & 0.4194 & 0.4191 & 0.4192 \\
1.222 & 0.4053 & 0.4049 & 0.4050 \\
1.396 & 0.3886 & 0.3881 & 0.3883 \\
1.571 & 0.3684 & 0.3686 & 0.3685 \\
\hline
\end{tabular}

\section{Results and Discussion}

In this section, representative numerical results are displayed with the help of graphical illustrations. Computations were carried out for various values of physical parameters such as the chemical reaction parameter $\gamma$, magnetic field parameter $M$, radiation parameter $R$, buoyancy ratio parameter $N$, Soret number $S r$, Dufour number $D u$, Prandtl number Pr and the Schmidt number $S c$. Figures 2-16 illustrate the influence of these material parameters on the velocity, temperature and the concentration profiles. In addition, Figures 17-27 illustrate the effects of the physical parameters on the local skin-friction coefficient and the rates of heat and mass transfer.

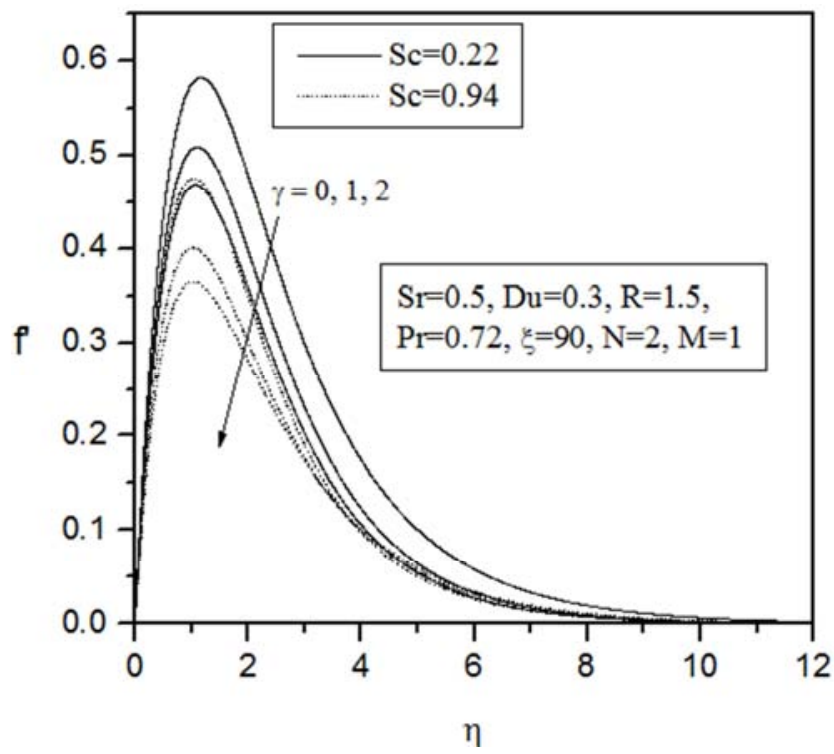

Figure 2. Effects of the chemical reaction parameter and the Schmidt number on the velocity profiles.

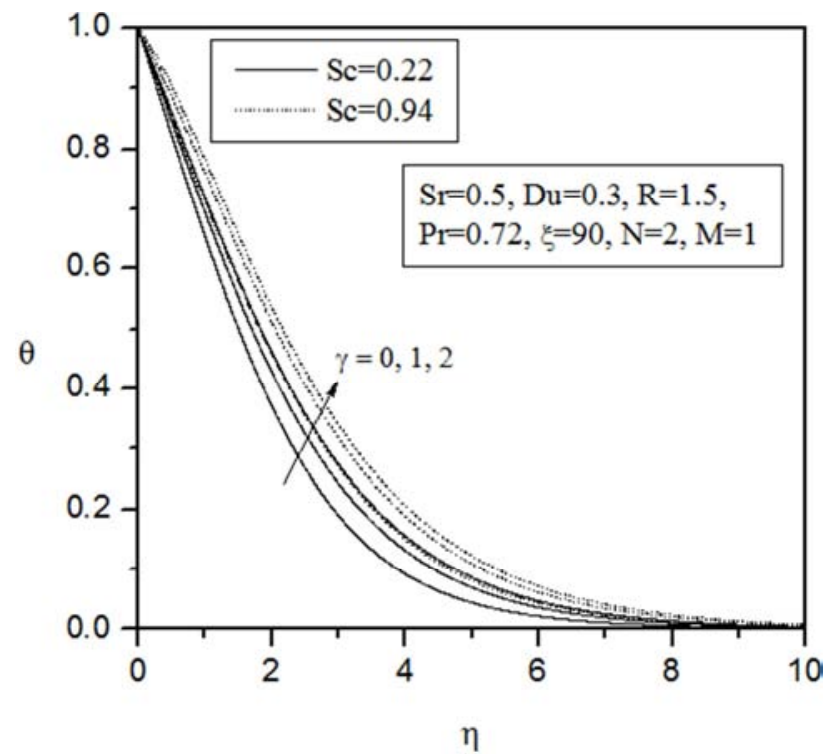

Figure 3. Effects of the chemical reaction parameter and the Schmidt number on the temperature profiles.

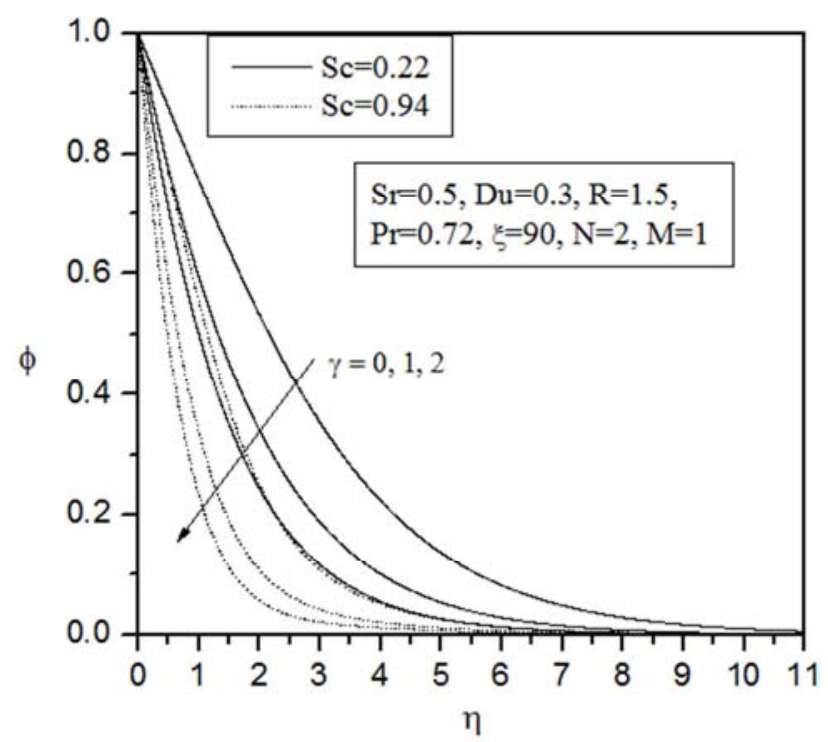

Figure 4. Effects of the chemical reaction parameter and the Schmidt number on the concentration profiles.

Figures 2-4 show the influence of the chemical reaction parameter $\gamma$ and the Schmidt number Sc on the velocity, temperature and concentration profiles in the boundary layer at the circumferential position $\xi=90^{\circ}$, respectively. Increasing the chemical reaction parameter produces a decrease in the species concentration. In turn, this causes the concentration buoyancy effects to decrease as $\gamma$ increases. Consequently, less flow is induced along the plate resulting in decreases in the fluid velocity in the boundary layer. Also, increasing the chemical reaction parameter leads to an increase in the temperature profiles. In addition, increasing the Schmidt number Sc causes decreases in both the velocity and concentration profiles while the temperature profiles increase. Furthermore, the concentration boundary layer thickness decreases as either $\gamma$ or Sc increases. These behaviors are clearly depicted in Figs. 2-4. 


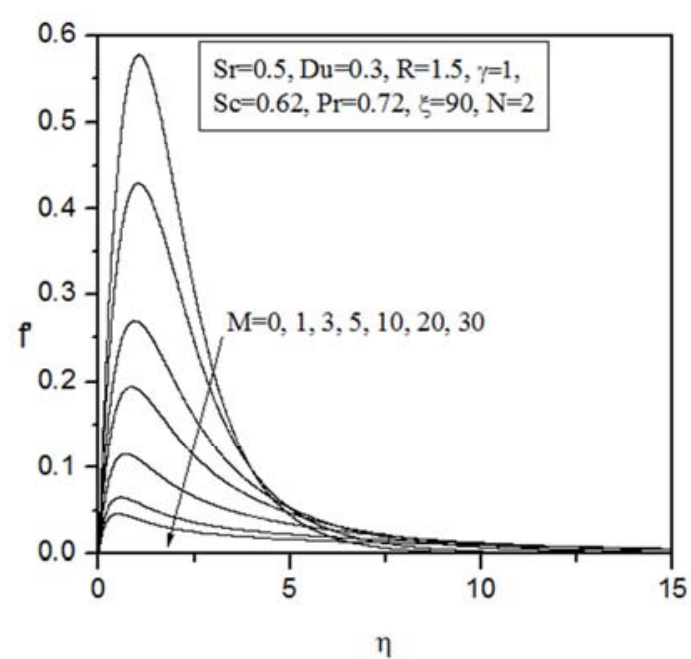

Figure 5. Effects of the magnetic field parameter on the velocity profiles.

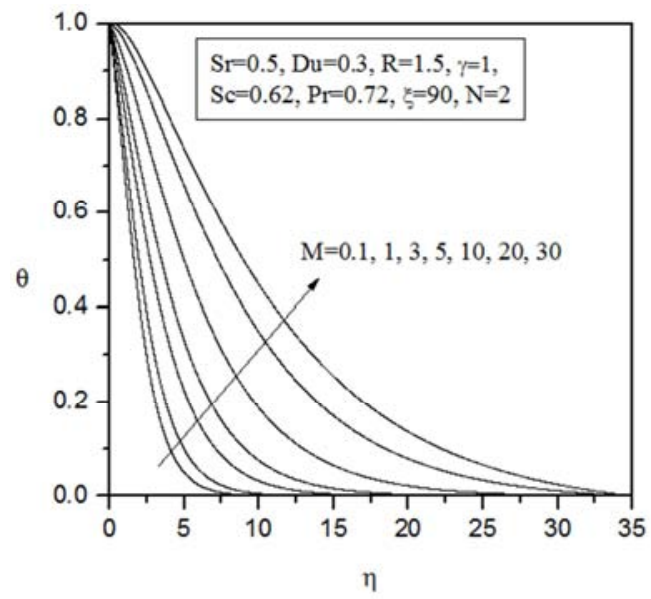

Figure 6. Effects of the magnetic field parameter on the temperature profiles.

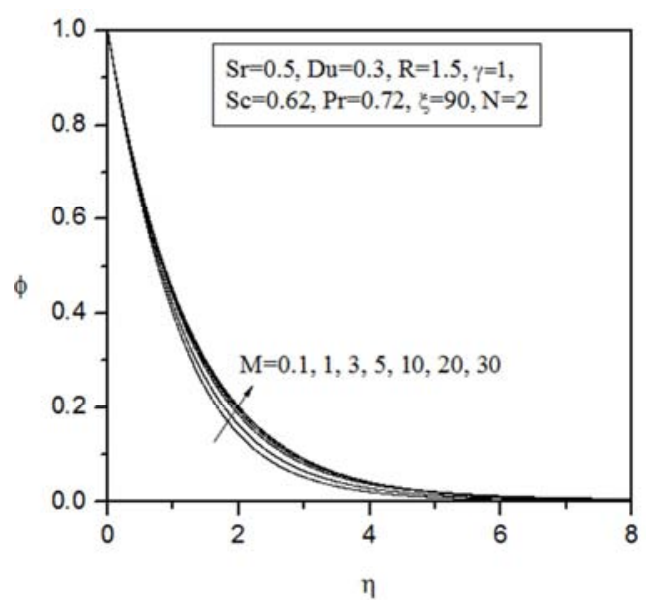

Figure 7. Effects of the magnetic field parameter on the concentration profiles.

Figures 5-7 present the effects of the magnetic field parameter $M$ on the velocity, temperature and concentration profiles at the circumferential position $\xi=90^{\circ}$, respectively. Application of a magnetic field has the tendency to slow down the movement of the fluid causing its velocity to decrease as the magnetic field parameter increases. In addition, this decrease in the flow movement as the magnetic field parameter increases is accompanied by increases on both the temperature and concentration profiles. These behaviors are clear from Figs. 5-7.

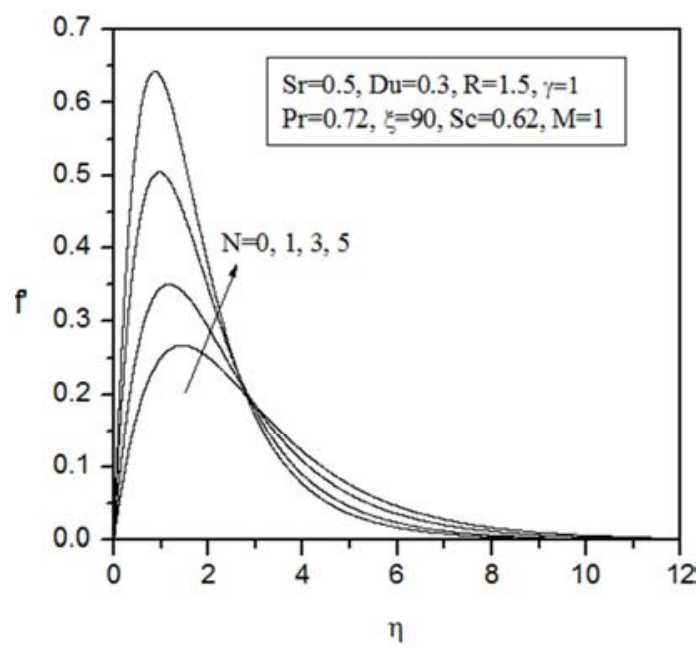

Figure 8. Effects of the buoyancy ratio on the velocity profiles.

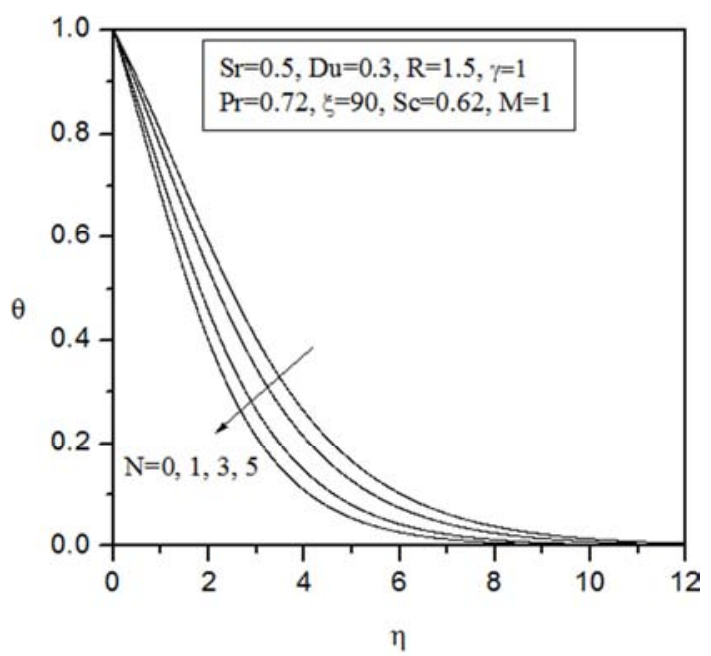

Figure 9. Effects of the buoyancy ratio on the temperature profiles.

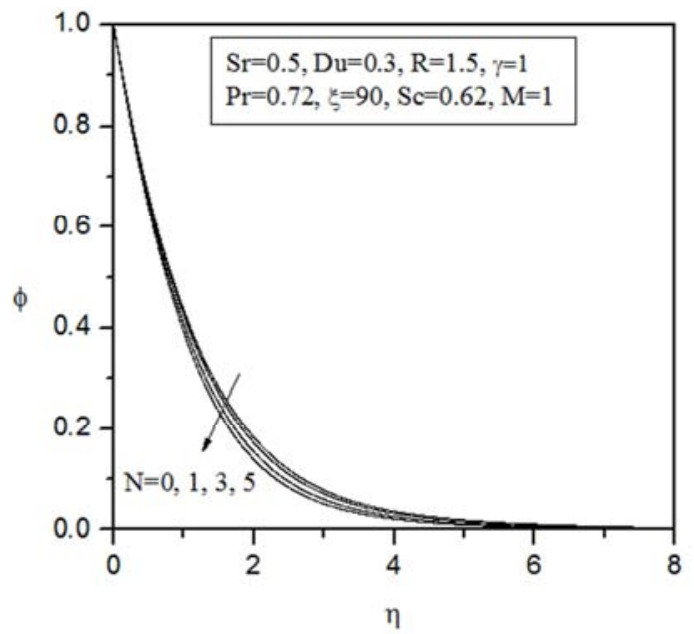

Figure 10. Effects of the buoyancy ratio on the concentration profiles. 
Figures 8-10 show the effects of the buoyancy ratio $\mathrm{N}$ on the velocity, temperature and concentration profiles at the circumferential position $\xi=90^{\circ}$, respectively. For $\mathrm{N}=0$ the flow around the sphere is induced only by thermal buoyancy effect due to temperature gradient. However, for $\mathrm{N}>0$ the flow around the sphere is caused by both temperature and concentration gradients. In this case, the flow will be aided by the concentration buoyancy effects. Therefore, as $\mathrm{N}$ increases, the flow around the sphere increases at the expense of decreased temperature and concentration. These decreases in both the temperature and concentration values are accompanied by corresponding decreases in both the thermal and solutal (concentration) boundary layer thicknesses. The increased flow is characterized by the formation of distinctive peaks in the velocity profiles in the immediate vicinity of the sphere surface as $\mathrm{N}$ increased. However, the velocity appear to decrease far downstream as $\mathrm{N}$ increases causing decreases in the hydrodynamic boundary layer thickness.

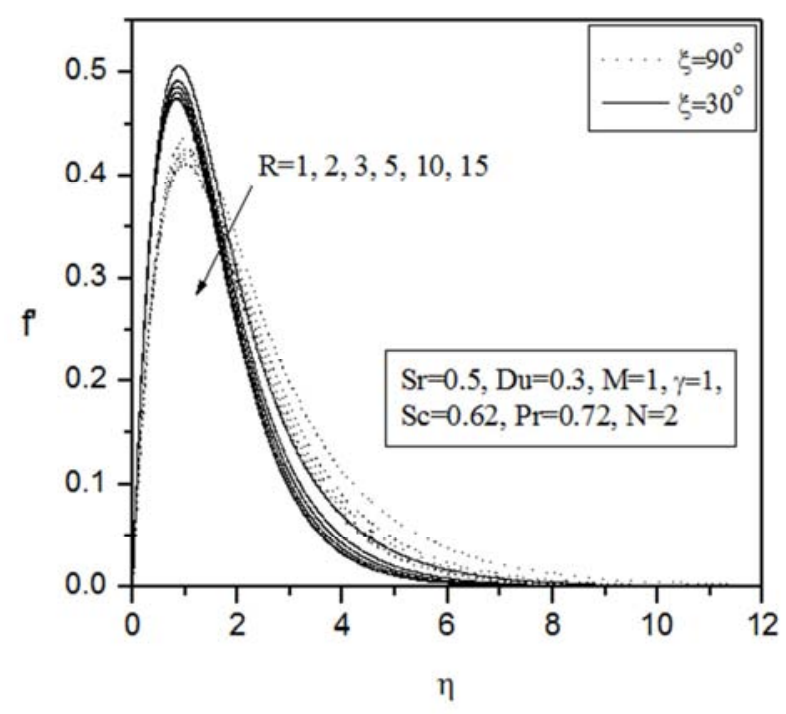

Figure 11. Effects of the radiation parameter on the velocity profiles.

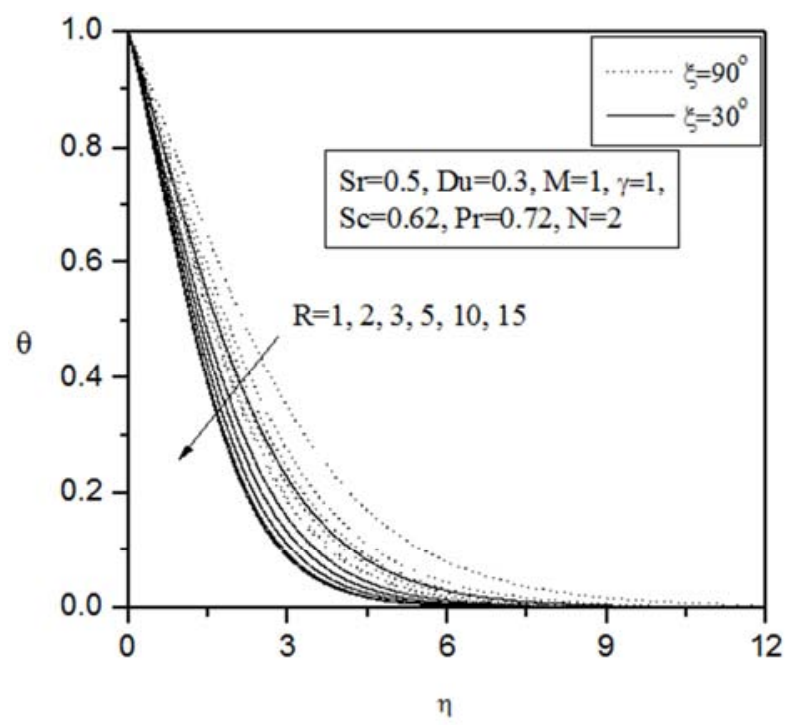

Figure 12. Effects of the radiation parameter on the temperature profiles.

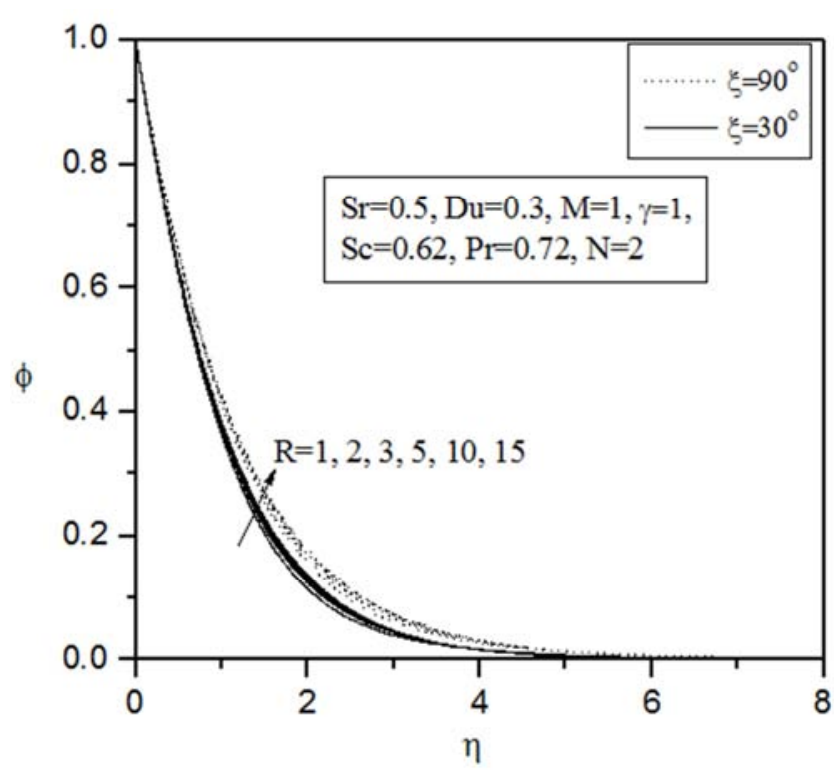

Figure 13. Effects of the radiation parameter on the concentration profiles.

Figures 11-13 display the effects of the thermal radiation parameter $R$ on the velocity, temperature and concentration profiles in the boundary layer at the circumferential position $\xi=90^{\circ}$, respectively. Decreasing the thermal radiation parameter $\mathrm{R}$ (i.e. increasing radiation effects) produces significant increase in the thermal state of the fluid causing its temperature to increase. This increase in the fluid temperature induces by virtue of the thermal buoyancy effect more flow in the boundary layer causing the velocity of the fluid there to increase. The concentration profiles tend to decrease as the radiation parameter decreases (i.e. increased radiation strength).

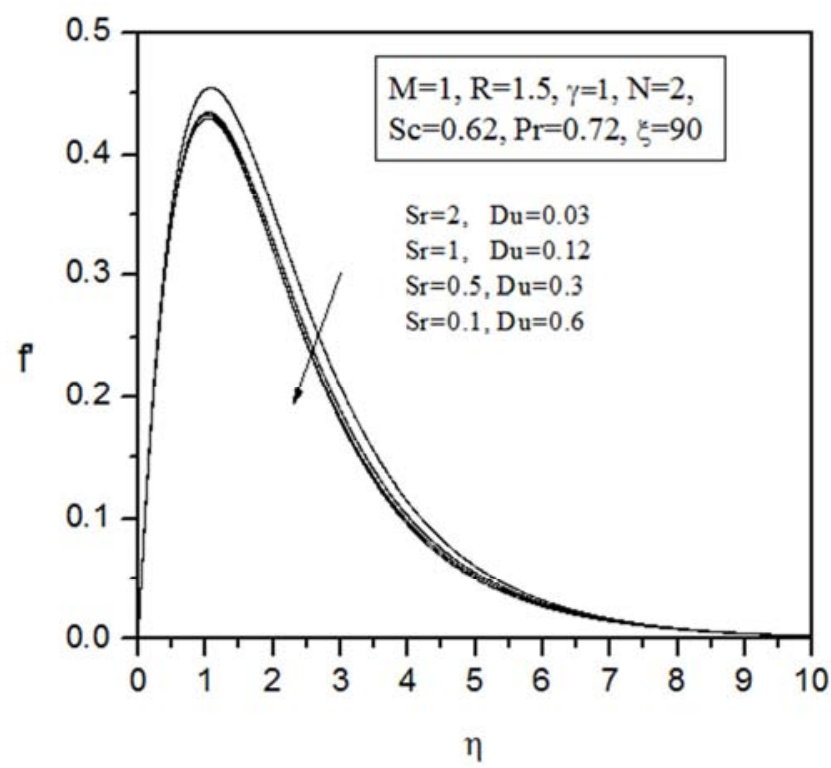

Figure 14. Effects of Dufour and Soret numbers on the velocity profiles. 


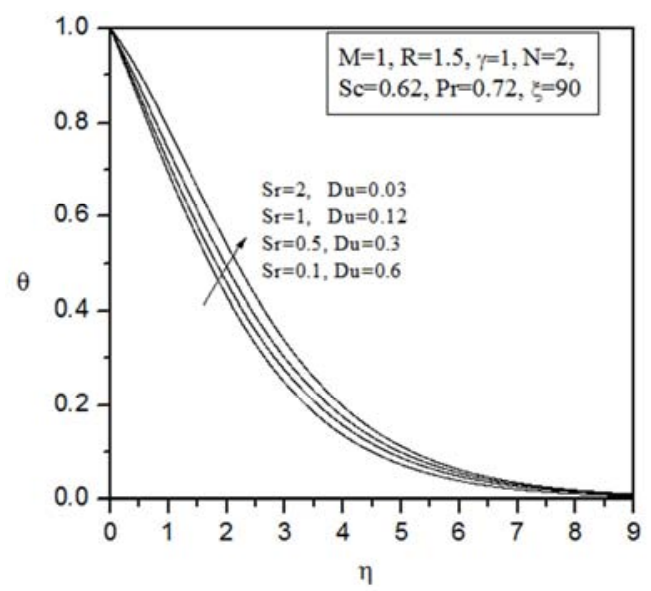

Figure 15. Effects of Dufour and Soret numbers on the temperature profiles.

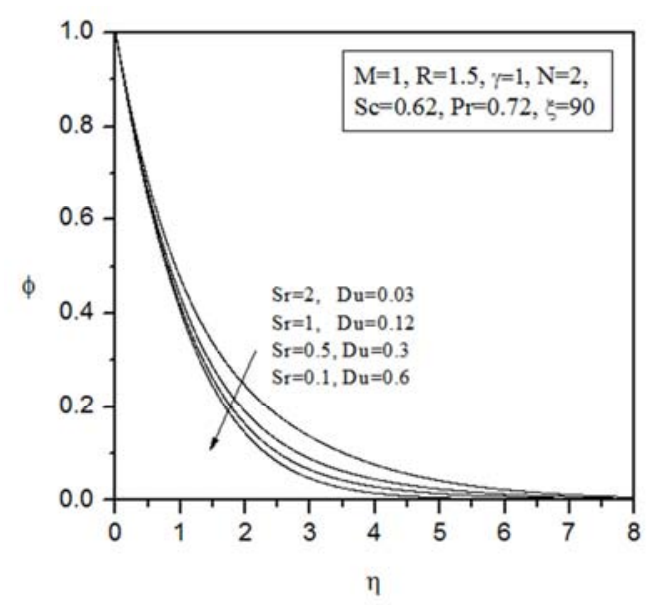

Figure 16. Effects of Dufour and Soret numbers on the concentration profiles.

Figures 14-16 display the effects of the combined Soret and Dufour numbers on the velocity, temperature and concentration profiles at the circumferential position $\xi=90^{\circ}$, respectively. As the Soret number decreases and the Dufour number increases, the fluid velocity and solute concentration profiles tend to decrease while the fluid temperature profiles increase.

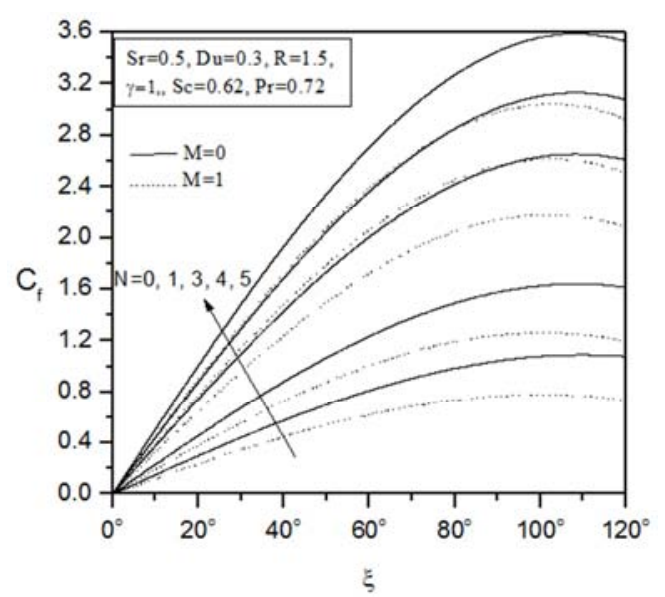

Figure 17. Effects of the buoyancy ratio and magnetic field parameter on the local skin-friction coefficient.

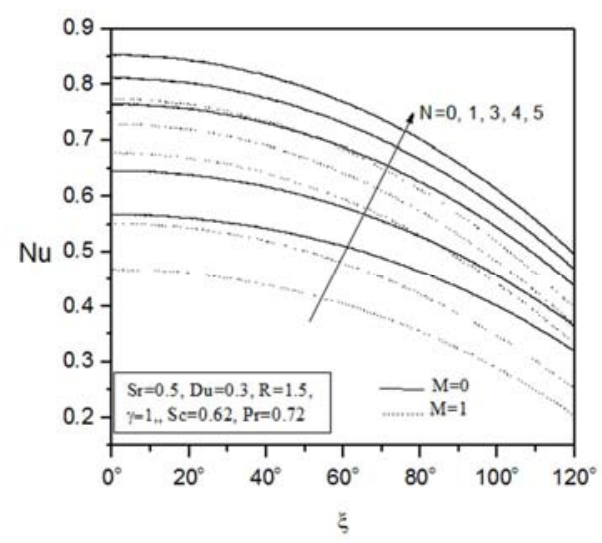

Figure 18. Effects of the buoyancy ratio and magnetic field parameter on the local heat transfer rate.

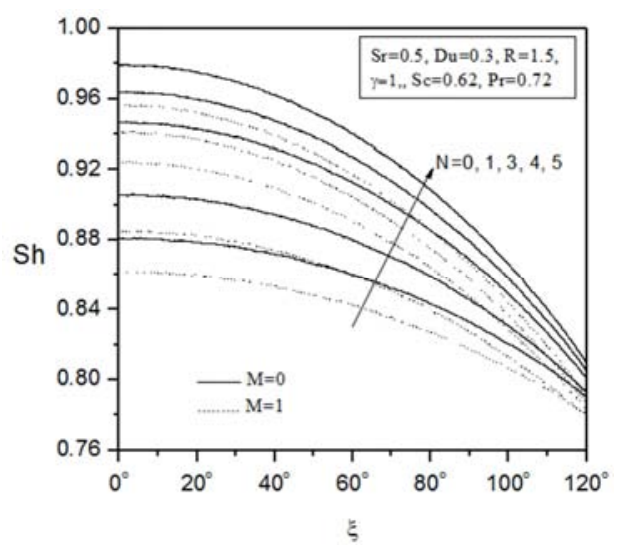

Figure 19. Effects of the buoyancy ratio and magnetic field parameter on the local mass transfer rate.

Figures 17-19 elucidate the effects of the buoyancy ratio $\mathrm{N}$ on the local skin-friction coefficient $\mathrm{C}_{\mathrm{f}}$ and the local rates of heat and mass transfer ( $\mathrm{Nu}$ and $\mathrm{Sh}$ ) along the sphere circumferential distance $\xi$ for two values of the magnetic field parameter $\mathrm{M}(\mathrm{M}=0,1)$, respectively. In general, in these figures and all subsequent figures, the values of $\mathrm{C}_{\mathrm{f}}$ increase (up to around $\xi=100^{\circ}$ ) while the values of $\mathrm{Nu}$ and $\mathrm{Sh}$ decrease with increasing values of $\xi$. It is predicted that all of $\mathrm{C}_{\mathrm{f}}, \mathrm{Nu}$ and $\mathrm{Sh}$ increase with increasing values of $\mathrm{N}$ while they decrease as the value of $\mathrm{M}$ increases.

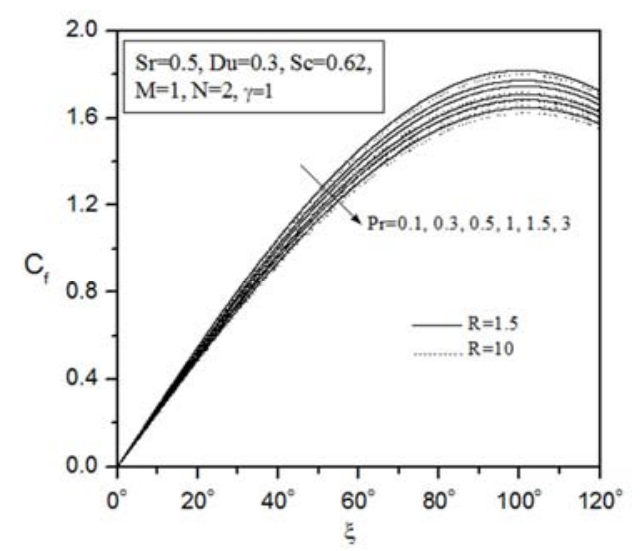

Figure 20. Effects of the Prandtl number and radiation parameter on the local skin-friction coefficient. 


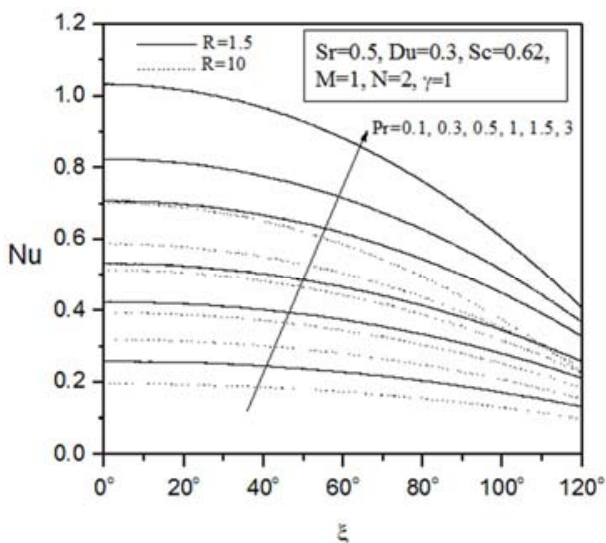

Figure 21. Effects of the Prandtl number and radiation parameter on the local heat transfer rate.

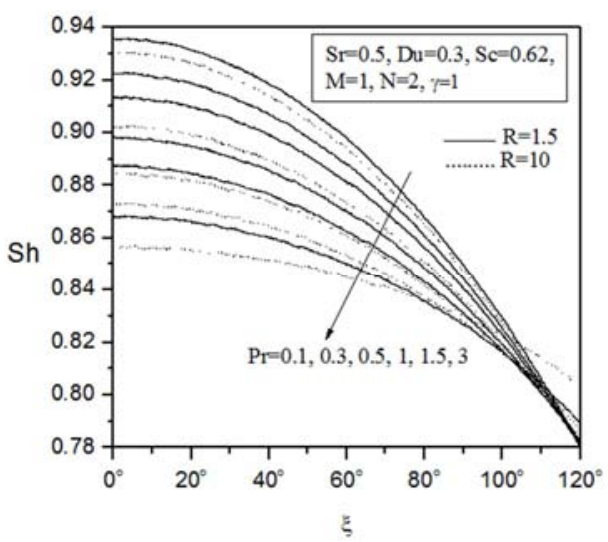

Figure 22. Effects of the Prandtl number and radiation parameter on the local mass transfer rate.

Figures 20-22 illustrate the effects of the Prandtl number Pr and the thermal radiation parameter $\mathrm{R}$ on the local skin-friction coefficient $\mathrm{C}_{\mathrm{f}}$ and the local rates of heat and mass transfer $(\mathrm{Nu}$ and Sh) along the sphere circumferential distance $\xi$ for two different values of the radiation parameter $\mathrm{R}$, respectively. It is clearly seen that the values of the local-skin friction coefficient and the local rate of mass transfer decrease while the values of the local rate of heat transfer increase as the Prandtl number increases. However, as the thermal radiation parameter $\mathrm{R}$ increases (which means decreased radiation effects), the values of $\mathrm{C}_{\mathrm{f}}, \mathrm{Nu}$ and $\mathrm{Sh}$ decrease.

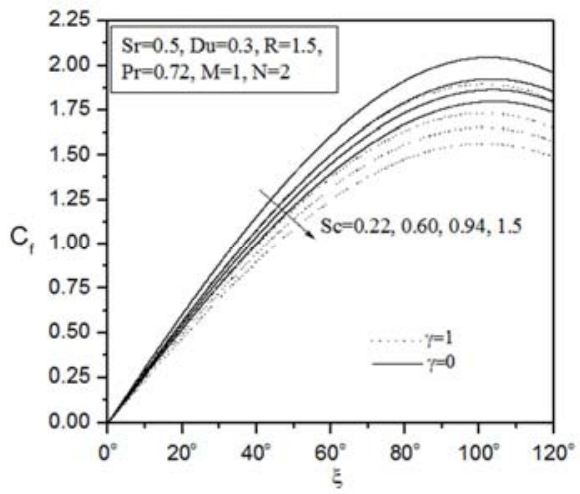

Figure 23. Effects of the Schmidt number and chemical reaction coefficient on the local skin-friction coefficient.

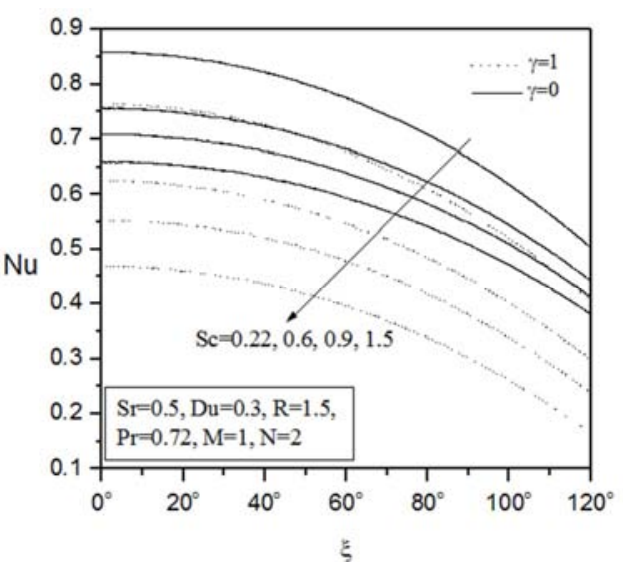

Figure 24. Effects of the Schmidt number and chemical reaction coefficient on the local heat transfer rate.

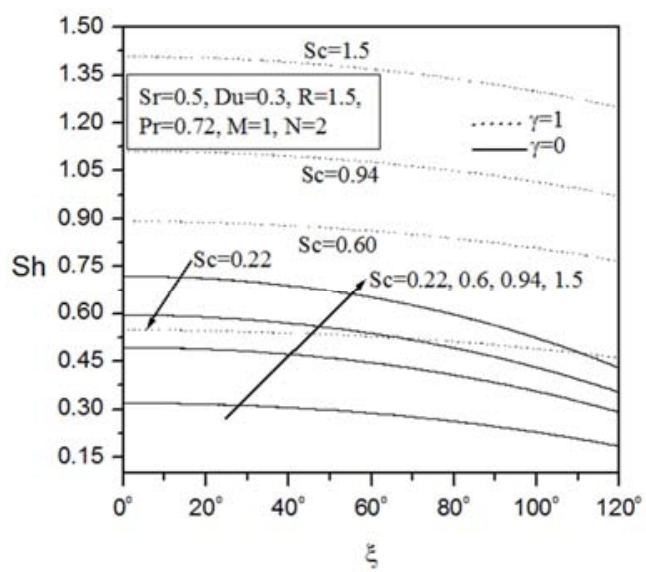

Figure 25. Effects of the Schmidt number and chemical reaction coefficient on the local mass transfer rate.

Figures 23-25 depict the influence of the Schmidt number Sc and the chemical reaction parameter $\gamma$ on the local skinfriction coefficient and local rates of heat and mass transfer for various values of $\xi$, respectively. It is observed that the local skin-friction coefficient and the local heat transfer rate decrease while local mass transfer rate increases as either of the Schmidt number or the chemical reaction parameter increases.

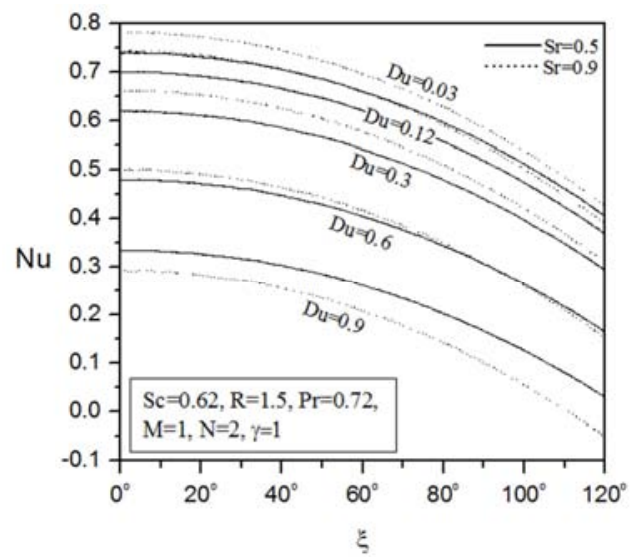

Figure 26. Effects of Dufour and Soret numbers on the local heat transfer rate. 


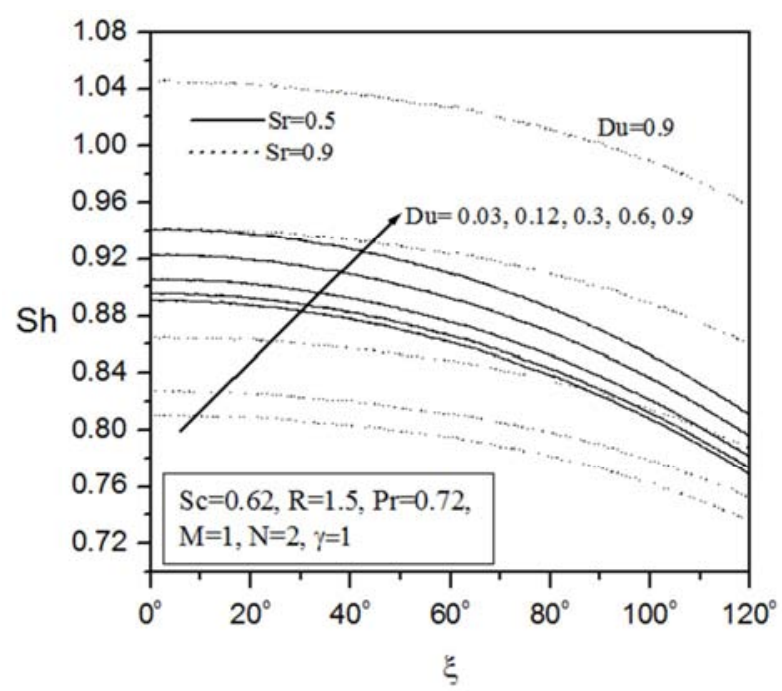

Figure 27. Effects of Dufour and Soret numbers on the local mass transfer rate.

Finally, Figs. 26 and 27 show the effects of Dufour number $\mathrm{Du}$ on the local rates of heat and mass transfer $(\mathrm{Nu}$ and $\mathrm{Sh})$ for two different values of the Soret number $\mathrm{Sr}(\mathrm{Sr}=0.5,0.9)$, respectively. It is predicted that local mass transfer rate increases while the local heat transfer rate decreases as the Dufour number Du increases for all values of $\xi$. However, the effect of the Soret number $\mathrm{Sr}$ on $\mathrm{Nu}$ and $\mathrm{Sh}$ depends on the value of $\mathrm{Du}$. For example, for $\mathrm{Du}>0.6$, the values of $\mathrm{Nu}$ are lower for $\mathrm{Sr}=0.9$ than for $\mathrm{Sr}=0.5$ for all values of $\xi$. On the other hand, for $\mathrm{Du}<0.6$, the values of $\mathrm{Nu}$ increase for all $\xi$ values as $\mathrm{Sr}$ is increased from 0.5 to 0.9 . For $\mathrm{Du}=0.6$, the values of $\mathrm{Nu}$ increase for $\xi<90^{\circ}$ and decrease for $\xi>90^{\circ}$ as $\mathrm{Sr}$ is increased from 0.5 to 0.9 . As for the mass transfer rate, it is predicted that the values of $\mathrm{Sh}$ increase for $\mathrm{Du}>0.3$ while they decrease for $\mathrm{Du}<0.3$ as $\mathrm{Sr}$ is increased from 0.5 to 0.9 for all values of $\xi$. For $\mathrm{Du}=0.3$, the values of $\mathrm{Sh}$ for $\mathrm{Sr}=0.9$ are lower than those for $\mathrm{Sr}=0.5$ for all values of $\xi<110^{\circ}$ while they are higher for $\xi>110^{\circ}$. All these features are clearly depicted in Figs. 26 and 27.

\section{Conclusions}

The problem of double diffusive natural convection MHD flow along a sphere was investigated in the presence of relevant physical effects such as homogeneous chemical reaction, thermal radiation and Soret and Dufour effects. Both the sphere wall temperature and concentration were assumed to be constant. The governing boundary-layer equations are formulated, non-dimensionalized and then transformed into a set of non-similarity equations which were solved numerically by an efficient, iterative, tri-diagonal, implicit finite-difference method. Comparisons with previously published work were made and the results were found to be in excellent agreement. It was found that, in general, the skin-friction coefficient increased as the buoyancy ratio or the Dufour number increased and it decreased as a result of increasing either of the magnetic field parameter, Prandtl number, Schmidt number, thermal radiation parameter or the chemical reaction parameter. In addition, the Nusselt number was predicted to increase due to increases in either of the buoyancy ratio or the Prandtl number while it decreased as either of the thermal radiation parameter, magnetic field parameter, Dufour number, chemical reaction parameter or the Schmidt number increased. Furthermore, the Sherwood number was predicted to increase as a result of increasing either of the buoyancy ratio, chemical reaction parameter, Dufour number or the Schmidt number while it decreased as a result of increasing either of the magnetic field parameter, thermal radiation parameter or the Prandtl number.

\section{References}

[1] S. P. Anjalidevi, R. Kandasamy, Effects of chemical reaction, heat and mass transfer on laminar flow along a semi infinite horizontal plate, Heat and Mass Transfer, 35 (6) (1999) 465467.

[2] S. P. Anjali Devi, R. Kandasamy, Effects of Chemical Reaction, Heat and Mass Transfer on MHD Flow past a Semi Infinite Plate, ZAMM - Journal of Applied Mathematics and Mechanics / Zeitschrift für Angewandte Mathematik und Mechanik, 80 (10) (2000) 697-700.

[3] A. Chamkha, M. A. Mansour, A. Aly, Unsteady MHD free convective heat and mass transfer from a vertical porous plate with Hall current, thermal radiation and chemical reaction effects, International Journal for Numerical Methods in Fluids, 65 (4) (2011) 432-447.

[4] A. Chamkha, A. Aly, M. Mansour, Similarity solution for unsteady heat and mass transfer from a stretching surface embedded in a porous medium with suction/injection and chemical reaction effects, Chemical Engineering Communications, 197 (6) (2010) 846-858.

[5] R. Muthucumaraswamy, P. Ganesan, On impulsive motion of a vertical plate with heat flux and diffusion of chemically reactive species, Forschung im Ingenieurwesen, 66 (1) (2000) 17-23.

[6] R. Muthucumaraswamy, P. Ganesan, First-order chemical reaction on flow past an impulsively started vertical plate with uniform heat and mass flux, Acta Mechanica, 147 (1) (2001) 45-57.

[7] H. S. Takhar, A. J. Chamkha, G. Nath, Flow and mass transfer on a stretching sheet with a magnetic field and chemically reactive species, International Journal of Engineering Science, 38 (12) (2000) 1303-1314.

[8] R. Muthucumaraswamy, Effects of a chemical reaction on a moving isothermal vertical surface with suction, Acta Mechanica, 155 (1) (2002) 65-70.

[9] J. A. Weaver, R. Viskanta, Natural convection due to horizontal temperature and concentration gradients-2. Species interdiffusion, Soret and Dufour effects, International Journal of Heat and Mass Transfer, 34 (12) (1991) 3121-3133.

[10] M. S. Malashetty, S. N. Gaikwad, Effect of cross diffusion on double diffusive convection in the presence of horizontal gradients, International Journal of Engineering Science, 40 (7) (2002) 773-787. 
[11] C. Soret, Influence de la temperature sur la distribution des sels dans leurs solutions,, C. R. Acad. Sci. Paris 91 (1880) 289-291.

[12] E. D, Analysis Of Heat And Mass Transfer, Taylor \& Francis, 1986.

[13] N. G. Kafoussias, E. W. Williams, Thermal-diffusion and diffusion-thermo effects on mixed free-forced convective and mass transfer boundary layer flow with temperature dependent viscosity, International Journal of Engineering Science, 33 (9) (1995) 1369-1384.

[14] M. Mansour, N. El-Anssary, A. Aly, Effects of chemical reaction and thermal stratification on MHD free convective heat and mass transfer over a vertical stretching surface embedded in a porous media considering Soret and Dufour numbers, Chemical engineering journal, 145 (2) (2008) 340345 .

[15] A. Chamkha, A. Aly, Heat and mass transfer in stagnationpoint flow of a polar fluid towards a stretching surface in porous media in the presence of Soret, Dufour and chemical reaction effects, Chemical Engineering Communications, 198 (2) (2010) 214-234.

[16] I. S. H. R. Grief, L. C. Lin, Laminar convection of a radiating gas in a vertical channel, J. Fluid Mech, 45 (1971) 513-520.

[17] W. G. V. A. C. Cogley, S. E. Gill, Differential approximation for radiative transfer in a non-gray-gas near equilibrium, AIAA J, 6 (1968) 551-553.

[18] M. M. Molla, M. Taher, M. M. Chowdhury, M. A. Hossain, Magnetohydrodynamic natural convection flow on a sphere in presence of heat generation, Nonlinear Analysis: Modelling and Control, 10 (4) (2005) 349-363.

[19] R. Nazar, N. Amin, Free convection boundary layer on an isothermal sphere in a micropolar fluid, International communications in heat and mass transfer, 29 (3) (2002) 377 386.

[20] R. Nazar, N. Amin, I. Pop, Free convection boundary layer on an isothermal horizontal circular cylinder in a micropolar fluid, Heat Transfer, 2 (2002) 525-530.

[21] M. Mansour, M. El-Hakiem, S. El Kabeir, Heat and mass transfer in magnetohydrodynamic flow of micropolar fluid on a circular cylinder with uniform heat and mass flux, Journal of Magnetism and Magnetic Materials, 220 (2) (2000) 259-270.
[22] M. M. Alam, M. Alim, M. M. Chowdhury, Viscous dissipation effects on MHD natural convection flow along a sphere, Journal of Mechanical Engineering, 36 (2006) 44-48.

[23] M.-J. Huang, Laminar free convection from a sphere with blowing and suction, Journal of heat transfer, 109 (2) (1987) 529-532.

[24] C.-Y. Cheng, Natural convection heat and mass transfer from a sphere in micropolar fluids with constant wall temperature and concentration, International Communications in Heat and Mass Transfer, 35 (6) (2008) 750-755.

[25] A. J. Chamkha, A. Rashad, A. M. Aly, Non-Darcy Natural Convection of a Nanofluid about a Permeable Vertical Cone Embedded in a Porous Medium, International Journal of Microscale and Nanoscale Thermal and Fluid Transport Phenomena, 4 (2) (2013) 99.

[26] A. Chamkha, A. M. Aly, M. Mansour, Effects of Chemical Reaction and Pressure Work on Free Convection over a Stretching Cone Embedded in a Porous Medium, International Journal of Industrial Mathematics, 4 (4) (2012) 319-333.

[27] A. Chamkha, A. M. Aly, Heat and Mass Transfer by Free Convective Flow of a Polar Fluid along a Sphere Embedded in a Porous Medium, Journal of Energy, Heat and Mass Transfer, 34 (2012) 19-47.

[28] A. J. Chamkha, A. Rashad, A. M. Aly, Transient natural convection flow of a nanofluid over a vertical cylinder, Meccanica, 48 (1) (2013) 71-81.

[29] N. A. Khan, F. Sultan, On the double diffusive convection flow of Eyring-Powell fluid due to cone through a porous medium with Soret and Dufour effects, AIP Advances, 5 (5) (2015) 057140.

[30] C. S. K. Raju, N. Sandeep, A. Malvandi, Free convective heat transfer of MHD Cu-kerosene nanofluid over a cone with temperature dependent viscosity, Acta Astronautica, 129 (2016) 419-428.

[31] E. Sparrow, R. Cess, Radiation Heat Transfer, 1978, Hemisphere, Wahington, DC.

[32] F. Blottner, Finite difference methods of solution of the boundary-layer equations, AIAA Journal, 8 (2) (1970) 193205. 